\title{
PURELY BAER MODULES AND PURELY RICKART MODULES
}

\author{
S. EBRAHIMI ATANI, M. KHORAMDEL, AND S. DOLATI PISHHESARI
}

Received 30 December, 2014

\begin{abstract}
We introduce the notions of purely Baer and purely Rickart modules. We provide several characterizations and investigate properties of each of these concepts. We provide new characterizations of several well-known classes of rings in terms of purely Baer and purely Rickart modules. It is shown that $R$ is a von Neumann regular ring iff every right $R$-module is purely Baer (purely Rickart). Also, we prove $R$ is left semihereditary iff every (finitely generated) free right $R$-module is purely Baer. Examples illustrating the results are presented.
\end{abstract}

2010 Mathematics Subject Classification: 16E50; 16E60; 16D80

Keywords: Baer modules, purely Baer modules, Rickart modules, purely Rickart modules

\section{INTRODUCTION}

The notions of Rickart and Baer rings have their roots in functional analysis, with close links to $C^{*}$-algebras and von Neumann algebras. Kaplansky introduced Baer rings to abstract various properties of $A W^{*}$-algebras and von Neumann algebras and complete $*$-regular rings in [11]. Motivated by Kaplansky's work on Baer rings, the notion of Rickart rings appeared in Maeda [15] and was further studied by Hattori [9] and other authors. A ring $R$ is called Baer (resp. right Rickart (or p.p.)) if the right annihilator of any nonempty subset (resp. any single element) of $R$ is generated by an idempotent, as a right ideal of $R$. It is well known that Baer rings and Rickart rings play an important role in providing a rich supply of idempotents and hence in the structure theory for rings.

Recently, the notions of Baer and Rickart rings and their generalizations were extended and studied in a general module-theoretic setting [1,13,14,16-18].

A module is called extending if every its submodule is essential in a direct summand. In [6], the notion of extending generalized to purely extending by replacing "direct summand" with "pure submodule". In [4], basic characterizations of purely extending modules are given.

Motivated by the notions of Baer and purely extending modules, we introduce the notion of purely Baer modules. In Section 3, we investigate purely Baer modules and give some results related to them. It is shown that a direct summand of a purely Baer module is purely Baer. Our focus, in this section, is on the question: When are (free) 
right $\mathrm{R}$-modules over a ring $R$ purely Baer? We obtain characterizations of wellknown classes of rings, in terms of free purely Baer modules over them. We show that the class of rings for which every (resp. free) module is purely Baer, is precisely that of (resp. left semihereditary) von Neumann regular rings. As an application of this, we prove that a commutative domain $R$ is Prüfer if and only if every free Rmodule is purely Baer. Some characterizations of right purely Baer rings are given. It is known that the definition of Baer rings is left-right symmetric. However we show that the definition of purely Baer rings is asymmetric.

In Section 4, the notion of purely Rickart modules is introduced. A characterization of von Neumann regular rings in terms of purely Rickart modules is given. It is shown that every free right $R$-module is purely Rickart if and only if every right ideal of $R$ is flat. We provide some characterizations of right purely Rickart rings. Also we show that the definition of purely Rickart rings is left-right symmetric, however the definition of Rickart rings is asymmetric.

\section{Preliminaries}

Throughout this paper, $R$ is an associative ring with identity and all modules are unitary. By $M_{R}$ (resp. ${ }_{R} M$ ), we denote a right (resp. left) $R$-module and $S=$ $\operatorname{End}\left(M_{R}\right)$ denotes the endomorphism ring of $M_{R} \cdot M^{I}$ (resp. $\left.M^{(I)}\right)$ stands for the direct product (resp. direct sum) of copies of $M$ indexed by a set $I$. Let $M$ be a right $R$-module, $I$ a subset of $R$ and $X$ a subset of $\operatorname{End}\left(M_{R}\right)$. We write $\mathrm{r}_{M}(X)=\{m \in$ $M: x m=0$ for all $x \in X\}$ and $\mathrm{r}_{R}(I)$ (resp. $\left.1_{R}(I)\right)=$ the right (resp. left) annihilator $I$ in $R$. Let $M$ be a module over a ring $R$. For submodules $N$ and $K$ of $M, N \leq K$ denotes $N$ is a submodule of $K$. I what follows, by $\leq^{\oplus}$, $\leq^{e s s}$ and $E(M)$ we denote, respectively, a module direct summand, an essential submodule and the injective hull of $M$. For a ring $R, \operatorname{Mat}_{n}(R)$ denotes the ring of $n \times n$ matrices over $R$.

In the following, we recall some known notions and facts needed in the sequel.

Definition 1. (1) A (short) exact sequence $\xi: 0 \rightarrow K \stackrel{\varphi}{\rightarrow} N \rightarrow M \rightarrow 0$ of right $R$ modules is said to be pure (exact) if $\xi \otimes_{R} T$ is an exact sequence (of abelian groups) for any left $R$-module $T$. In this case, we say that $\varphi(K)$ is a pure submodule of $N$ (see [12] and [19]). It is clear that every direct summand is a pure submodule.

(2) A module $M$ over a ring $R$ will be called finitely presented if there exists an exact sequence $0 \rightarrow K \rightarrow F \rightarrow M \rightarrow 0$ of $R$-modules, where $F$ is free and both $F$ and $K$ are finitely generated (see [12] and [19]).

(3) A right module $M$ over a ring $R$ is called divisible provided $M x=M$ for all regular elements $x \in R$ (see [8]).

(4) A right $R$-module $M$ is called pure injective, if $M$ is injective with respect to every pure exact sequence of right $R$-modules. A ring $R$ is called right pure semisimple if every right $R$-module is pure injective (see [22]). 
(5) A module $M_{R}$ is called $F P$-injective (or absolutely pure) if, for any finitely generated submodule $K$ of a free right $R$-module $F$, every homomorphism $K \rightarrow M$ extends to a map $F \rightarrow M$ (see [22]).

(6) A module $M_{R}$ is called regular, provided that each of its submodule is pure (see [5]).

(7) A right $R$-module $M$ is said to be Baer (resp. Rickart), if for any left ideal $I$ of $\operatorname{End}(M)($ resp. $\phi \in \operatorname{End}(M)), \mathrm{r}_{M}(I)\left(\operatorname{resp} . \mathrm{r}_{M}(\phi)\right)$ is a direct summand of $M$ (see [17], [18], [13] and [14]).

(8) A module $M$ is called purely extending if every submodule of $M$ is essential in a pure submodule of $M$. Equivalently, every closed submodule is a pure submodule (see [4]).

(9) A module is called torsionless if it can be embedded in a direct product of copies of the base ring (see [12]).

We refer to [12] and [22] for the undefined notions in this article.

Proposition 1 ( [12, Corollary 4.86]). Let $\xi: 0 \rightarrow K \rightarrow F \rightarrow M \rightarrow 0$ be an exact sequence of $R$-modules with $F$ flat. Then $M$ is flat if and only if $\xi$ is pure.

Proposition 2 ( [21, Lemma 2.2]). Let $R$ be a ring and $0 \rightarrow K \rightarrow P \rightarrow M \rightarrow 0$ an exact sequence of right $R$-modules with $P$ projective. Then the following statements are equivalent:

(1) $M$ is flat;

(2) Given any $x \in K$, there exists a homomorphism $g: P \rightarrow K$ such that $g(x)=x$;

(3) Given any $x_{1}, \ldots, x_{n}$ in $K$, there exists a homomorphism $g: P \rightarrow K$ such that $g\left(x_{i}\right)=x_{i}$ for $i=1,2, \ldots, n$.

Proposition 3 ( [2, Theorem 4.1] ). For any ring $R$, the following statements are equivalent:

(1) $R$ is left semi-hereditary;

(2) Every right ideal of $R$ is flat, and the direct product of an arbitrary family of copies of $R$ is flat as a right $R$-module;

(2) Every torsion-less right $R$-module is flat.

\section{Purely Baer modules}

In this section, we investigate connections between purely Baer modules and various existing concepts and obtain some of their useful properties. Examples of purely Baer modules include semisimple modules, regular modules, Baer modules, nonsingular purely extending modules and modules over von Neumann regular rings.

Definition 2. Let $M$ be a right $R$-module and $S=\operatorname{End}_{R}(M)$. Then $M$ is called a purely Baer module, if $\mathrm{r}_{M}(I)$ is a pure submodule of $M$ for each left ideal $I$ of $S$.

A ring $R$ is called (resp. left) right purely Baer, if (resp. $\left.1_{R}(I)\right) \mathrm{r}_{R}(I)$ is a pure (resp. left) right ideal of $R$ for each (resp. right) left ideal $I$ of $R$. 
First, we characterize right purely Baer rings in terms of cyclic torsionless modules.

Theorem 1. Let $R$ be a ring. Then $R$ is right purely Baer if and only if every cyclic torsionless right $R$-module is flat.

Proof. It is known that a cyclic right $R$-module $R / I$ is torsionless if and only if $I=\mathrm{r}_{R}(X)$ for some subset $X$ of $R$. Assume that $R$ is right purely Baer. Let $M$ be a cyclic torsionless right $R$-module. As $M \cong R / I$ for some right ideal $I$ of $R$, $I=\mathrm{r}_{R}(X)$ for some $X \subseteq R$. Since $R$ is right purely Baer, $I=\mathrm{r}_{R}(J)$ is pure in $R_{R}$, where $J$ is a left ideal generated by $X$. Thus $R / I$ is flat by Proposition 1 and so $M$ is flat.

Conversely, assume that every cyclic torsionless right $R$-module is flat. Let $I$ be a left ideal of $R$. Then $R / \mathrm{r}_{R}(I)$ is torsionless and so is flat. Hence $\mathrm{r}_{R}(I)$ is pure by Proposition 1 . Thus $R$ is right purely Baer.

In the following, it is shown that direct summands of a purely Baer module are purely Baer.

Proposition 4. Let $M$ be a purely Baer module with $S=\operatorname{End}(M)$. Then every direct summand of $M$ is purely Baer.

Proof. Let $M$ be a purely Baer module and $N \leq^{\oplus} M$ say $M=N \oplus K$ for some $K \leq M$. Let $J$ be a left ideal of $S^{\prime}=\operatorname{End}(N)$. Set $I=\left\{\left.\psi \oplus 0\right|_{K}: \psi \in J\right\}$. Then $\mathrm{r}_{M}(S I)$ is a pure submodule of $M$, because $M$ is purely Baer. As $K \subseteq \mathrm{r}_{M}(S I)$, $\mathrm{r}_{M}(S I)=\mathrm{r}_{M}(S I) \cap N \oplus K$. An inspection shows that $\mathrm{r}_{M}(S I) \cap N=\mathrm{r}_{N}(J)$. Since $\mathrm{r}_{N}(J) \leq{ }^{\oplus} \mathrm{r}_{M}(S I), \mathrm{r}_{N}(J)$ is pure in $\mathrm{r}_{M}(S I)$. Therefore by transitivity of the pure submodules property, $\mathrm{r}_{N}(J)$ is pure in $M$. Hence $\mathrm{r}_{N}(J)$ is pure in $N$. Therefore $N$ is purely Baer.

In the following, it is shown that the notions of purely Baer modules and Baer modules coincide for finitely generated flat modules over a right noetherian ring and torsion free injective modules over a semiprime right Goldie ring.

Theorem 2. (1) Let $R$ be a right noetherian ring and $M$ a finitely generated flat right $R$-module. Then $M$ is purely Baer if and only if it is Baer.

(2) Let $M$ be a torsion free injective right $R$-module over a semiprime right Goldie ring $R$. Then $M$ is purely Baer if and only if it is Baer.

(3) Let $R$ be a right pure semisimple ring. Then a right $R$-module $M$ is purely Baer if and only if $M$ is Baer.

Proof. (1) Let $M$ be a purely Baer module and $I$ a left ideal of $S=\operatorname{End}\left(M_{R}\right)$. Then $\mathrm{r}_{M}(I)$ is pure in $M$. Hence by Proposition $1, M / \mathrm{r}_{M}(I)$ is flat. As $M / \mathrm{r}_{M}(I)$ is finitely generated and $R$ is right noetherian, $M / \mathrm{r}_{M}(I)$ is finitely presented by [12, Theorem 4.29]. Hence $M / \mathrm{r}_{M}(I)$ is projective by [12, Theorem 4.30]. This implies that $\mathrm{r}_{M}(I) \leq{ }^{\oplus} M$. Hence $M$ is a Baer module. The converse is clear. 
(2) Let $M$ be a purely Baer module and $I$ a left ideal of $S=\operatorname{End}\left(M_{R}\right)$. Then $\mathrm{r}_{M}(I)$ is pure in $M$. Hence by $[22,34.8]$, for each regular element $x \in R$,

$$
\mathrm{r}_{M}(I) x=\mathrm{r}_{M}(I) \cap M x=\mathrm{r}_{M}(I) \cap M=\mathrm{r}_{M}(I),
$$

because $M$ is divisible. Hence $\mathrm{r}_{M}(I)$ is divisible. Therefore [7, Theorem 7.11] implies that $\mathrm{r}_{M}(I)$ is injective. Hence $\mathrm{r}_{M}(I) \leq^{\oplus} M$. So $M$ is Baer. The converse is clear.

(3) If $R$ is right pure semisimple, then every pure exact sequence splits. Hence $M$ is purely Baer if and only if it is Baer.

Theorem 3. Let $M$ be a nonsingular right $R$-module. If $M$ is purely extending, then $M$ is a purely Baer module.

Proof. Let $M$ be a purely extending module and $I$ a left ideal of $\operatorname{End}_{R}(M)$. Then $\mathrm{r}_{M}(I) \leq{ }^{e s s} T$ for some pure submodule $T$ of $M$. Let $t \in T$. Then $J=\{r \in R: t r \in$ $\left.\mathrm{r}_{M}(I)\right\}$ is an essential right ideal of $R_{R}$. Thus $t J \subseteq \mathrm{r}_{M}(I)$ and so for each $f \in I$, $f(t J)=f(t) J=0$. As $M$ is nonsingular, $f(t)=0$ for each $f \in I$. Therefore $\mathrm{r}_{M}(I)=T$ is a pure submodule of $M$ and it implies that $M$ is a purely Baer module.

The next example shows that the converse of Theorem 3 is not true.

Example 1. [4] Let

$$
R=\left(\begin{array}{ccc}
F & 0 & F \\
0 & F & F \\
0 & 0 & F
\end{array}\right),
$$

the $F$-subalgebra of the ring of $3 \times 3$ matrices over a field $F$. This ring is left and right artinian hereditary (so it is left and right semihereditary). Hence $R$ is left and right nonsingular. Hence by Theorem 5, $R$ is right purely Baer. We show $R$ is not purely extending. Let $I$ be a closed right ideal of $R$. Then $I$ is finitely generated (because $R$ is noetherian) and so $R / I$ is finitely presented. If $I$ is pure, then $R / I$ is flat by Proposition 1 . As $R / I$ is finitely presented, $R / I$ is projective by [12, Theorem 4.30]. Hence $I \leq{ }^{\oplus} R_{R}$. This implies that if $R_{R}$ is purely extending, then $R_{R}$ is extending. However $R$ is not right extending (see [4] and [3, Example 5.5 ]).

Next, we characterize the class of rings $R$ for which every right R-module is purely Baer as precisely that of the von Neumann regular rings.

Theorem 4. The following are equivalent for a ring $R$.

(1) Every right $R$-module is purely Baer;

(2) Every purely extending right $R$-module is purely Baer;

(3) Every extending right $R$-module is purely Baer;

(4) Every injective right $R$-module is purely Baer;

(5) Every right $R$-module is FP-injective;

(6) $R$ is von Neumann regular. 
Proof. (1) $\Rightarrow(2),(2) \Rightarrow$ (3) and (3) $\Rightarrow$ (4) are clear.

(4) $\Rightarrow(5)$ Let $M$ be an arbitrary right $R$-module. Then by (4), $\mathrm{E}(M) \oplus \mathrm{E}(\mathrm{E}(M) / M)$ is purely Baer. Let $\psi: \mathrm{E}(M) \rightarrow \mathrm{E}(\mathrm{E}(M) / M)$ be defined by $\psi(x)=x+M$ for each $x \in \mathrm{E}(M)$. We can extend $\psi$ to an endomorphism $\bar{\psi}$ of $\mathrm{E}(M) \oplus \mathrm{E}(\mathrm{E}(M) / M)$ and $\operatorname{Ker}(\bar{\psi})=M$. Hence $M$ is pure in $\mathrm{E}(M) \oplus \mathrm{E}(\mathrm{E}(M) / M)$. This implies that $M$ is pure in $\mathrm{E}(\boldsymbol{M})$. Hence $M$ is FP-injective by $[22,35.8]$.

$(5) \Rightarrow(6)$ By $[22,37.6], R$ is a von Neumann regular ring.

(6) $\Rightarrow$ (1) By [20, Proposition 3.18], every right $R$-module is flat. Let $M$ be a right $R$-module and $I$ a left ideal of $\operatorname{End}(M)$. By (6), $M$ and $M / \mathrm{r}_{M}(I)$ are flat modules. This implies that $\mathrm{r}_{M}(I)$ is pure in $M$ by Proposition 1 .

The following examples exhibit purely Baer modules which are not Baer.

Example 2. Let

$$
R=\left(\begin{array}{cc}
\prod_{i=1}^{\infty} F_{i} & \oplus_{i=1}^{\infty} F_{i} \\
\oplus_{i=1}^{\infty} F_{i} & <\oplus_{i=1}^{\infty} F_{i}, 1>
\end{array}\right),
$$

where $F_{i}=F$ is a field for each $i \in \mathbb{N}$ and $<\oplus_{i=1}^{\infty} F_{i}, 1>$ is the $F$-algebra generated by $\oplus_{i=1}^{\infty} F_{i}$ and 1 . Then $R$ is a von Neumann regular ring. Hence by Theorem $4, R_{R}$ is a purely Baer module. However, it is not a Baer module by [13, Example 2.19].

Example 3. Let $F$ be a field and $V$ be an infinite dimensional vector space over $F$. Set $J=\left\{x \in \operatorname{End}_{F}(V) \mid \operatorname{dim}_{F}(x V)<\infty\right\}$ and $R=F+J$. By [7, Example 6.19], $\mathrm{R}$ is regular. Therefore $M=R \oplus R / J$ is purely Baer by Theorem 4 . However, $M$ is not Baer. If $M$ is Baer, then $M$ is Rickart and so $J \leq^{\oplus} R$ by [13, Proposition 2.24], a contradiction (because $J=\operatorname{Soc}\left(R_{R}\right)$ and $J$ is essential in $R_{R}$ (see [7]).

Proposition 5. Let $P$ be a finitely generated projective right $R$-module. Then $P$ is a purely Baer module if and only if $S=\operatorname{End}(P)$ is a right purely Baer ring.

Proof. Let $P=x_{1} R+\ldots+x_{n} R\left(x_{i} \in P\right)$ be a purely Baer projective module and $I$ be a left ideal of $S$. We show $\mathrm{r}_{S}(I)$ is a pure right ideal of $S$. Let $f \in$ $\mathrm{r}_{S}(I)$. Then $f\left(x_{i}\right) \in \mathrm{r}_{P}(I)$ for each $1 \leq i \leq n$. As $P$ is purely Baer, $\mathrm{r}_{P}(I)$ is a pure submodule. Hence $P / \mathrm{r}_{P}(I)$ is flat by Proposition 1 and so by Proposition 2, there exists a homomorphism $\psi: P \rightarrow \mathrm{r}_{P}(I)$ such that $\psi\left(f\left(x_{i}\right)\right)=f\left(x_{i}\right)$ for each $1 \leq i \leq n$. Hence $\psi f=f$. We can take $\psi$ as an endomorphism of $P$. Since $\psi(P) \subseteq \mathrm{r}_{P}(I), \psi \in \mathrm{r}_{S}(I)$. Now let $\phi: S \rightarrow \mathrm{r}_{S}(I)$ be defined by $\phi(h)=\psi h$ for each $h \in S$. Then $\phi(f)=\psi f=f$. Therefore by Proposition 2, $S / \mathrm{r}_{S}(I)$ is a flat right $S$-module. Hence by Proposition $1, \mathrm{r}_{S}(I)$ is a pure right ideal of $S$. Thus $S$ is right purely Baer.

Conversely, assume that $P$ is a finitely generated projective right $R$-module and $S=\operatorname{End}\left(P_{R}\right)$ a right purely Baer ring. We show $P$ is purely Baer. Since $P$ is finitely generated and projective, $P \leq{ }^{\oplus} R^{n}$ for some positive integer $n$. As $\operatorname{End}\left(R^{n}\right) \cong$ $\operatorname{Mat}_{n}(R)$, there exists $E^{2}=E \in \operatorname{Mat}_{n}(R)$ such that $P=E R^{n}$. Hence $S=E \operatorname{Mat}_{n}(R) E$. 
Let $I$ be a left ideal of $S$ and $b=\left(\begin{array}{c}b_{1} \\ b_{2} \\ \vdots \\ b_{n}\end{array}\right) \in \mathrm{r}_{P}(I)$. Then an inspection shows that

$$
B=\left(\begin{array}{cccc}
b_{1} & b_{1} & \cdots & b_{1} \\
b_{2} & b_{2} & \cdots & b_{2} \\
\vdots & \vdots & \ddots & \vdots \\
b_{n} & b_{n} & \cdots & b_{n}
\end{array}\right) \in \mathrm{r}_{S}(I)
$$

As $b \in P, E b=b$. Thus $E B=B$. This implies that $B \in S$, because $B h=E B h \in$ $E R^{n}$ for each $h \in R^{n}$. Since $\mathrm{r}_{S}(I)$ is a pure right ideal in $S, S / \mathrm{r}_{S}(I)$ is a flat right $S$-module by Proposition 1. Hence by Proposition 2, there exists a homomorphism $\theta: S \rightarrow \mathrm{r}_{S}(I)$ such that $\theta(B)=B$. We can take $\theta$ as a right endomorphism of $S$. Hence $\theta(B)=\theta(1) B=B$, where 1 is identity element of $S$ and $\theta(1) \in \mathrm{r}_{S}(I)$. Let $\theta(1)=A=\left(\begin{array}{cccc}a_{11} & a_{12} & \cdots & a_{1 n} \\ a_{21} & a_{22} & \cdots & a_{2 n} \\ \vdots & \vdots & \ddots & \vdots \\ a_{n 1} & a_{n 2} & \cdots & a_{n n}\end{array}\right)$. As $A B=B, \sum_{j=1}^{n} a_{i j} b_{j}=b_{i}$ for each $1 \leq i \leq n$. Hence $A b=b$. As $A \in \mathrm{r}_{S}(I)$, we can define $\beta: P \rightarrow \mathrm{r}_{P}(I)$ by $f(p)=A p$ for each $p \in P$. Since $A \in \mathrm{r}_{S}(I), A p \in \mathrm{r}_{P}(I)$ for each $p \in P$. Also $A b=b$ implies that $\beta(b)=A b=b$. Therefore by Proposition $2, P / \mathrm{r}_{P}(I)$ is a flat right $R$-module and so by Proposition $1, \mathrm{r}_{P}(I)$ is pure in $P$. Hence $P$ is purely Baer.

Next, we characterize rings $R$ for which every (finitely generated) free right $R$ module is purely Baer. We show that these are precisely the left semihereditary rings.

Theorem 5. The following are equivalent for a ring $R$ :

(1) Every free right $R$-module is purely Baer;

(2) Every projective right $R$-module is purely Baer;

(3) Every finitely generated free right $R$-module is purely Baer;

(4) Every finitely generated projective right $R$-module is purely Baer;

(5) Every finitely generated torsionless right $R$-module is flat;

(6) Every torsionless right $R$-module is flat;

(7) $R$ is a left semihereditary ring.

Proof. (1) $\Rightarrow$ (2) and (3) $\Rightarrow$ (4) are clear from Proposition 4.

(2) $\Rightarrow$ (3) is clear.

(4) $\Rightarrow$ (5) Let $M$ be a finitely generated torsionless right $R$-module. Hence $M \subseteq$ $R^{I}$ for some index set $I$. As $M$ is finitely generated, there exists an epimorphism $\psi: F \rightarrow M$, where $F$ is a finitely generated free right $R$-module. By (4), $F$ is purely Baer. It can be shown that $\operatorname{Ker}(\psi)=\cap_{i \in I} \operatorname{Ker}\left(\pi_{i} \psi\right)$, where $\pi_{i}$ is the canonical projection from $R^{I}$ onto its $i$ th coordinates. We can take $\pi_{i} \psi$ as an endomorphism 
of $F$. Hence $\operatorname{Ker}(\psi)=\mathrm{r}_{M}(J)$, where $J$ is a left ideal of $S=\operatorname{End}(F)$ generated by the set $\left\{\pi_{i} \psi\right\}_{i \in I}$. Since $F$ is purely $\operatorname{Baer} \operatorname{Ker}(\psi)$ is a pure submodule of $F$. Therefore $M \cong F / \operatorname{Ker}(\psi)$ is flat by Proposition 1 .

$(5) \Rightarrow(6)$ As every submodule of a torsionless right $R$ is torsionless, every finitely generated submodule of a torsionless right $R$-module is flat. Hence every torsionless right $R$-module is flat (it is known that, a module is flat provided that every of its finitely generated submodule is flat).

(6) $\Rightarrow(7)$ is from Proposition 3.

(7) $\Rightarrow$ (1) Let $F$ be a free right $R$-module and $I$ a left ideal of $S=\operatorname{End}(F)$. As for each $\psi \in S, F / \operatorname{Ker}(\psi) \cong \operatorname{Im}(\psi), F / \operatorname{Ker}(\psi)$ is a torsionless module. Hence $\prod_{\psi \in I} F / \operatorname{Ker}(\psi)$ is torsionless, because direct product of torsionless modules is torsionless. Let $\phi: F / \bigcap_{\psi \in I} \operatorname{Ker}(\psi) \rightarrow \prod_{\psi \in I} F / \operatorname{Ker}(\psi)$ be defined by $\phi(x+$ $\bigcap_{\psi \in I} \operatorname{Ker}(\psi)=(x+\operatorname{Ker}(\psi))_{\psi \in I}$ for each $x \in F$. It is clear that $\phi$ is a monomorphism. Thus $F / \bigcap_{\psi \in I} \operatorname{Ker}(\psi)$ is torsionless. Therefore by Proposition 3,

$F / \bigcap_{\psi \in I} \operatorname{Ker}(\psi)$ is flat. Hence $\mathrm{r}_{F}(I)=\bigcap_{\psi \in I} \operatorname{Ker}(\psi)$ is pure in $F$ by Proposition 1 . Thus $F$ is a purely Baer module.

Corollary 1. The following are equivalent for a ring $R$.

(1) $R$ is left semihereditary;

(2) For each $n>1, \operatorname{Mat}_{n}(R)$ is a right purely Baer ring.

Proof. By Theorem 5, $R$ is left semihereditary if and only if $R^{n}$ is purely Baer right $R$-module if and only if $\operatorname{Mat}_{n}(R)$ is right purely Baer for each $n$, by Proposition 5.

Remark 1. By Theorem 5, every finitely generated free right $R$-module is purely Baer if and only if every free right $R$-module is purely Baer. But it is not true for the class of Baer modules. Let $R$ be a commutative Prüfer domain which is not a Dedekind domain (hereditary). Then every finitely generated free right $R$-module is Baer by [18, Theorem 3.9]. However there is a free right $R$-module that is not Baer, by [18, Theorem 3.3].

The following example gives an example of a module $M$ such that every submodule of $M$ is purely Baer.

Example 4. Let

$$
R=\left(\begin{array}{ll}
\mathbb{Z} & \mathbb{Q} \\
0 & \mathbb{Q}
\end{array}\right) .
$$

By [12, Example 2.33], $R$ is right hereditary and left semihereditary. Let $M=(e R)^{n}$, where $e$ is an arbitrary idempotent of $R$ and $n \in \mathbb{N}$. Since $R$ is right noetherian and right hereditary, every submodule of $M$ is finitely generated and projective. Therefore by Theorem 5, every submodule of $M$ is purely Baer. Also, for each submodule $N$ of $M, \operatorname{End}(N)$ is a right purely Baer ring, by Proposition 5. 
The next example shows that the definition of purely Baer rings is not left-right symmetric. As we know, the definition of Baer rings is left-right symmetric.

Example 5. Let

$$
T=\left(\begin{array}{cc}
S / I & S / I \\
0 & S
\end{array}\right)
$$

where $S$ is a von Neumann ring and $I$ a right ideal of $S$ which is not a direct summand of $S_{S}$. Then by [12, Example 2.34], $T$ is left semihereditary but not right semihereditary. By Corollary 1, there exists positive integer $n$ such that $\operatorname{Mat}_{n}(T)$ is a right purely Baer ring which is not left purely Baer ring.

As a consequence of Theorem 5, we provide a characterization of Prüfer domains in terms of the purely Baer property for (finitely generated) free (projective) modules.

Theorem 6. For any commutative domain $R$, the following are equivalent:

(1) Every free $R$-module is purely extending;

(2) Every free R-module is purely Baer;

(3) Every finitely generated free R-module is purely Baer;

(4) $R$ is a Prüfer domain.

Proof. (1) $\Rightarrow$ (2) Since $R$ is nonsingular, every free $R$-module is nonsingular. Therefore every free $R$-module is purely Baer by Theorem 3 .

(2) $\Rightarrow(3)$ is clear.

(3) $\Rightarrow(4)$ It is known that a commutative domain is semihereditary if and only if it is a Prüfer domain by [12, Theorem 4.69]. Hence the result is clear by Theorem 5 .

(4) $\Rightarrow$ (1) [4, Proposition 2.1]

\section{Purely Rickart modules}

Motivated by the definitions of Rickart modules, we introduce the notions of purely Rickart modules and relatively purely Rickart modules and collect some basic properties of these classes of modules. Examples of purely Rickart modules include semisimple modules, Rickart modules, purely Baer modules and modules over regular rings. We begin with the key definition of this section.

Definition 3. Let $M$ be a right $R$-module and $S=\operatorname{End}\left(M_{R}\right)$. Then $M$ is called a purely Rickart module, if $\mathrm{r}_{M}(\psi)=\operatorname{Ker}(\psi)$ is a pure submodule of $M$ for each $\psi \in S$.

A ring $R$ is called (resp. left) right purely Rickart, if (resp. $\left.1_{R}(a)\right) \mathrm{r}_{R}(a)$ is a pure (resp. left) right ideal of $R$ for each $a \in R$.

It is known that the definition of Rickart rings is asymmetric. However the following shows that the definition of purely Rickart rings is symmetric. Also purely Rickart rings are known as P.F rings [10]. 
Theorem 7. The following are equivalent for a ring $R$ :

(1) $R$ is right purely Rickart;

(2) Every principle right ideal of $R$ is flat;

(3) Every principle left ideal of $R$ is flat;

(4) $R$ is left purely Rickart.

Proof. (1) $\Leftrightarrow(2)$ and (3) $\Rightarrow$ (4) Let $a \in R$. Then (resp. $R a) a R$ is flat as a (resp. left) right $R$-module if and only if (resp. $\left.1_{R}(a)\right) \mathrm{r}_{R}(a)$ is a pure (resp. left) right ideal of $R$ by Proposition 1 .

(2) $\Leftrightarrow$ (3) By [10].

One can easily show that under the following conditions, the notions of Rickart module and purely Rickart module coincide, as in the proof of Theorem 2.

Proposition 6. (1) Let $R$ be a right noetherian ring and $M$ a finitely generated flat right $R$-module. Then $M$ is purely Rickart if and only if it is Rickart.

(2) Let $M$ be a torsion free injective right $R$-module over a semiprime right Goldie ring $R$. Then $M$ is purely Rickart if and only if it is Rickart.

(3) Let $R$ be a right pure semisimple ring and $M$ a right $R$-module. Then $M$ is purely Rickart if and only if it is Rickart.

Definition 4. A module $M$ is called $N$-purely Rickart, if $\operatorname{Ker}(\phi)$ is a pure submodule of $M$ for every homomorphism $\phi: M \rightarrow N$.

In view of the above definition, a right $R$-module $M$ is purely Rickart if and only if $M$ is $M$-purely Rickart.

The following propositions are useful to prove our main theorems.

Proposition 7. (1) Let $M$ and $N$ be right $R$-modules. Then $M$ is any $N$-purely Rickart if and only if for any direct summand $K \leq{ }^{\oplus} M$ and any submodule $L \leq N$, $K$ is L-purely Rickart.

(2) The following are equivalent for a right $R$-module $M$ :

(i) $M$ is purely Rickart;

(ii) For any submodule $N \leq M$ and any direct summand $K \leq{ }^{\oplus} M, K$ is $N$-purely Rickart;

(iii) For any $K, N \leq^{\oplus} M$, and $\phi \in \operatorname{Hom}_{R}(M, N)$, $\operatorname{Ker}\left(\left.\phi\right|_{K}\right)$ is pure in $K$.

(3) Every direct summand of a purely Rickart module is a purely Rickart module.

Proof. (1) Suppose that $M$ is any $N$-purely Rickart. Let $K=e M$ for some idempotent element $e$ of $\operatorname{End}\left(M_{R}\right)$ and $\phi: K \rightarrow L$ be a homomorphism. Then $\phi e$ is a homomorphism from $M$ to $N$. Hence $\operatorname{Ker}(\phi e)$ is pure in $M$. As $\operatorname{Ker}(\phi e)=$ $\operatorname{Ker}(\phi) \oplus(1-e) M, \operatorname{Ker}(\phi)$ is pure in $\operatorname{Ker}(\phi e)$. This implies that $\operatorname{Ker}(\phi)$ is pure in $M$ and so is in $N$. Thus $K$ is $L$-purely Rickart. The converse is clear.

(2) and (3) are clear from (1). 
We now characterize the von Neumann regular rings in terms of purely Rickart modules.

Theorem 8. The following are equivalent for a ring $R$ :

(1) Every right $R$-module is purely Baer;

(2) Every right $R$-module is purely Rickart;

(3) Every pure injective right $R$-module is injective;

(4) $R$ is von Neumann regular.

Proof. (1) $\Rightarrow$ (2) is clear.

(2) $\Rightarrow$ (3) Let $M$ be a pure injective right $R$-module. By (2), $E(M) \oplus E(E(M) / M)$ is purely Rickart. It implies that $E(M)$ is $E(E(M) / M)$-purely Rickart by Proposition 7. Therefore $\operatorname{Ker}(\pi)=M$ is pure in $E(M)$, where $\pi: E(M) \rightarrow E(E(M) / M)$ is natural homomorphism. Since $M$ is pure injective, the pure exact sequence $0 \rightarrow$ $M \rightarrow E(M) \rightarrow E(M) / M \rightarrow 0$ splits. Hence $M \leq^{\oplus} E(M)$. Thus $M$ is injective.

(3) $\Rightarrow$ (4) By $[22,37.6]$

(4) $\Rightarrow$ (1) By Theorem 4.

It is clear that every Rickart module is purely Rickart. The following examples exhibit purely Rickart modules which are not Rickart.

Example 6. Let $R=\prod_{i=1}^{\infty} F_{i}$, where $F_{i}=F$ is a field for each $i \in \mathbb{N}$ and $I=\oplus_{i=1}^{\infty} F_{i}$. Then by Theorem $8, M=R \oplus R / I$ is a purely Rickart right $R$ module, which is not a Rickart module (if $M$ is a Rickart module then $I \leq{ }^{\oplus} R$ by [13, Proposition 2.24] which is a contradiction).

Example 7. Let $S=\prod_{i=1}^{\infty} \mathbb{Z}_{2}$. Consider

$$
R=\left\{\left(a_{n}\right)_{n=1}^{\infty} \in S \mid a_{n} \text { is eventually constant }\right\},
$$

a subring of $S$. Then $R$ is a von Neumann regular ring. Hence by Theorem 8, $R \oplus E(R)$ is a purely Rickart module which is not a Rickart module by [14, Example 2.18].

In the next theorem, we characterize the rings $R$ for which every free $R$-module is purely Rickart.

Theorem 9. The following are equivalent for a ring $R$ :

(1) Every free right $R$-module is purely Rickart;

(2) Every projective right $R$-module is purely Rickart;

(3) Every finitely generated free right $R$-module is purely Rickart;

(4) Every finitely generated projective right $R$-module is purely Rickart;

(5) For each $n>1, \operatorname{Mat}_{n}(R)$ is a right purely Rickart ring;

(6) Every right ideal of $R$ is flat;

(7) Every submodule of a flat right $R$-module is flat.

Since condition (6) is left-right symmetric, the left-handed versions of (1), (2), (3), (4), (5) and (7) also hold. 
Proof. (1) $\Rightarrow$ (2) and (3) $\Rightarrow$ (4) are clear by Proposition 7.

$(2) \Rightarrow(3)$ is clear.

$(5) \Rightarrow(6)$ Assume that $\operatorname{Mat}_{n}(R)$ is a right purely Rickart ring for any $n>1$. Let $I=a_{1} R+\ldots+a_{n} R$ be a finitely generated right ideal of $R$ and $A \in \operatorname{Mat}_{n}(R)$ with first row $\left(a_{1}, a_{2}, \ldots, a_{n}\right)$, and zero elsewhere. Then $\mathrm{r}_{\mathrm{Mat}_{n}(R)}\left(\operatorname{Mat}_{n}(R) A\right)$ is a pure right ideal of $\operatorname{Mat}_{n}(R)$. Hence $A \operatorname{Mat}_{n}(R)$ is a flat right $\operatorname{Mat}_{n}(R)$-module by Proposition 1. Since $R$ and $\operatorname{Mat}_{n}(R)$ are Morita equivalent and flatness is preserved across Morita equivalences, $\left(A \operatorname{Mat}_{n}(R)\right)_{R} \cong I_{R}^{n}$ implies that $I$ is a flat right $R$ module. Hence every finitely generated right ideal of $R$ is flat. So every right ideal of $R$ is flat.

(6) $\Rightarrow(5)$ Let $A \in \operatorname{Mat}_{n}(R)$. As $\operatorname{Mat}_{n}(R)$ is a flat right $R$-module, $A \operatorname{Mat}_{n}(R)$ is a flat right $R$-module. Hence $A \operatorname{Mat}_{n}(R)$ is a flat right $\operatorname{Mat}_{n}(R)$-module by a similar argument as in above. Hence $\operatorname{Mat}_{n}(R)$ is right purely Rickart by Theorem 7 .

(4) $\Rightarrow(6)$ Let $I$ be a finitely generated right ideal of $R$. Then $I$ is homomorphic image of a finitely generated free right $R$-module $F$. Hence there exists an epimorphism $\varphi: F \rightarrow I$. We can take $\varphi$ as an endomorphism $F$. Hence $\mathrm{r}_{F}(\varphi)$ is a pure submodule of $F$. Thus by Proposition $1, F / \mathrm{r}_{F}(\varphi) \cong I$ is a flat $R$-module. Hence every finitely generated right ideal of $R$ is flat. So every right ideal of $R$ is flat.

(6) $\Rightarrow$ (7) By [12, Lemma 4.66], every submodule of a flat right $R$-module is flat.

(7) $\Rightarrow(1)$ Let $F$ be a free right $R$-module and $\psi \in \operatorname{End}(F)$. Then $M / \operatorname{Ker}(\psi) \cong$ $\operatorname{Im}(\psi) \leq F$ implies that $M / \operatorname{Ker}(\psi)$ is flat by (7). Hence $\operatorname{Ker}(\psi)$ is a pure submodule of $F$. Hence $F$ is a purely Rickart module.

Remark 2. By Theorem 9, every finitely generated free right $R$-module is purely Rickart if and only if every free right $R$-module is purely Rickart. However, the similar result for the class of Rickart modules does not hold true. Let $R$ be right semihereditary ring which is not hereditary. Then by [14, Theorem 3.6], every finitely generated free right $R$-module is Rickart. However there is a free right $R$-module that is not Rickart, by [14, Theorem 3.5].

The next example shows that the class of purely Rickart modules properly contains the class of purely Baer modules.

Example 8. Let

$$
R=\left(\begin{array}{cc}
S & S / I \\
0 & S / I
\end{array}\right)
$$

where $S$ is a von Neumann ring and $I$ a right ideal of $S$ which is not a direct summand of $S_{S}$. Then by [12, Example 2.34], $R$ is right semihereditary but not left semihereditary. Since $R$ is right semihereditary, every right ideal of $R$ is flat. Hence by Theorem 9, every free right $R$-module is purely Rickart. As $R$ is not left semihereditary, there is a free right $R$-module which is not purely Baer by Theorem 5 . Hence there exists a free $R$-module $F$ which is purely Rickart but not purely Baer. 
The following example proves the existence of a module $M$ such that $M^{n}$ is a purely Rickart (purely Baer) module, but $M^{n+1}$ is not purely Rickart (purely Baer). The following example is due to Jøndrup (see [10], [14, Example 3.15] and [18, Example 3.17]).

Example 9. Let $n$ be any natural number, $K$ be any commutative field, and let $R$ be the $K$-algebra on the $2(n+1)$ generators $X_{i}, Y_{i}(i=1, \ldots, n+1)$ with the defining relation

$$
\sum_{i=1}^{n+1} X_{i} Y_{i}=0 .
$$

As $R^{n}$ is a Baer module by [18, Example 3.17], $R^{n}$ is purely Rickart (purely Baer). But $R^{n+1}$ is not purely Rickart (purely Baer). Otherwise, if $R^{n+1}$ is purely Rickart (purely Baer), then one can show that every $(n+1)$ generated ideal of $R$ is flat, as in the proof of Theorem $9((4) \Rightarrow(6))$. However, it is proved that there exists an $(n+1)$ generated ideal which is not flat (see [10]). Hence $R^{n+1}$ is not purely Rickart (purely Baer).

\section{ACKNOWLEDGEMENT}

The authors are deeply indebted to the referee for many helpful comments and suggestions for the improvement of this article.

\section{REFERENCES}

[1] S. Atani, M. Khoramdel, and S. P. Hesari, "T-Rickart modules." Colloq. Math., vol. 128, no. 1, pp. 87-100, 2012, doi: 10.4064/cm128-1-8.

[2] S. U. Chase, "Direct products of modules.” Trans. Am. Math. Soc., vol. 97, pp. 457-473, 1961, doi: $10.2307 / 1993382$.

[3] A. Chatters and C. Hajarnavis, "Rings in which every complement right ideal is a direct summand." Q. J. Math., Oxf. II. Ser., vol. 28, pp. 61-80, 1977, doi: 10.1093/qmath/28.1.61.

[4] J. Clark, "On purely extending modules." in Abelian groups and modules. Proceedings of the international conference in Dublin, Ireland, August 10-14, 1998. Basel: Birkhäuser, 1999, pp. 353-358.

[5] D. Fieldhouse, "Regular modules over semi-local rings.” Rings, Modules, Radicals. Colloquia Math. Soc. Janos Bolyai 6, 193-196., 1973.

[6] L. Fuchs, "Notes on generalized continuous modules.” 1995.

[7] K. R. Goodearl, Von Neumann regular rings., 1979.

[8] K. R. Goodearl and R. Warfield, An introduction to noncommutative Noetherian rings. 2nd ed., 2nd ed. Cambridge: Cambridge University Press, 2004.

[9] A. Hattori, "A foundation of torsion theory for modules over general rings." Nagoya Math. J., vol. 17, pp. 147-158, 1960, doi: 10.1017/S0027763000002099.

[10] S. Jøndrup, "P.P. rings and finitely generated flat ideals." Proc. Am. Math. Soc., vol. 28, pp. 431495, 1971, doi: 10.2307/2037986.

[11] I. Kaplansky, "Rings of operators." Mathematics Lecture Note Series. New York-Amsterdam: W.A. Benjamin, Inc. VI, 151 p. (1968)., 1968.

[12] T. Lam, Lectures on modules and rings. New York, NY: Springer, 1999. 
[13] G. Lee, S. Rizvi, and C. S. Roman, "Rickart modules." Commun. Algebra, vol. 38, no. 11, pp. 4005-4027, 2010, doi: 10.1080/00927872.2010.507232.

[14] G. Lee, S. Rizvi, and C. S. Roman, "Direct sums of Rickart modules." J. Algebra, vol. 353, no. 1, pp. 62-78, 2012, doi: 10.1016/j.jalgebra.2011.12.003.

[15] S. Maeda, "On a ring whose principal right ideals generated by idempotens form a lattice." J. Sci. Hiroshima Univ., Ser. A, vol. 24, pp. 509-525, 1960.

[16] L. Mao, "Baer endomorphism rings and envelopes." J. Algebra Appl., vol. 9, no. 3, pp. 365-381, 2010, doi: 10.1142/S0219498810003963.

[17] S. Rizvi and C. S. Roman, "Baer and quasi-Baer modules." Commun. Algebra, vol. 32, no. 1, pp. 103-123, 2004, doi: 10.1081/AGB-120027854.

[18] S. Rizvi and C. S. Roman, "On direct sums of Baer modules." J. Algebra, vol. 321, no. 2, pp. 682-696, 2009, doi: 10.1016/j.jalgebra.2008.10.002.

[19] D. Simson, "On pure semi-simple Grothendieck categories. I." Fundam. Math., vol. 100, pp. 211 222, 1978.

[20] B. Stenström, "Rings of quotients. An introduction to methods of ring theory." Die Grundlehren der mathematischen Wissenschaften. Band 217. Berlin-Heidelberg-New York: Springer-Verlag. VIII, 309 p. DM 92.00., 1975.

[21] R. Ware, "Endomorphism rings of projective modules." Trans. Am. Math. Soc., vol. 155, pp. 233 256, 1971, doi: 10.2307/1995475.

[22] R. Wisbauer, Foundations of module and ring theory. , a handbook for study and research ed. Philadelphia etc.: Gordon and Breach Science Publishers, 1991.

Authors' addresses

S. Ebrahimi Atani

University of Guilan, Department of Mathematics, P.O.Box 1914, Rasht, Iran

E-mail address: ebrahimi@guilan.ac.ir

M. Khoramdel

University of Guilan, Department of Mathematics, P.O.Box 1914, Rasht, Iran

E-mail address: mehdikhoramdelegmail.com

S. Dolati Pishhesari

University of Guilan, Department of Mathematics, P.O.Box 1914, Rasht, Iran

E-mail address: saboura-dolati@yahoo.com 